\title{
Min 基因突变小鼠模型在肠道肿瘤研究中的应用
}

盛弘强, 陈俭, 来茂德

浙江大学医学院病理学与病理生理学系, 杭州 310058

摘要: 迄今为止, 肠道肿瘤相关的基因突变小鼠或敲除小鼠大约有 30 多种, Min(multiple intestinal neoplasia)小 鼠是具有肠道多发性腺瘤特征的A $\mathrm{A}$ 基因突变小鼠, 被认为是当前较为理想的家族性腺瘤性息肉病(FAP)的研 究模型。APC 基因是Wnt 途径中重要的抑癌基因, 该途径不仅是动物胚胎发育过程中关键的信号转导途径, 也 对结直肠肿瘤的发生发展起到不同寻常的作用。对 Min 小鼠模型历史、分子遗传学与表型特征、肠道肿瘤与 Wnt 途径异常、抑癌基因甲基化、TGF- $\beta$ 途径和多药耐药基因等内容进行了介绍, 并分析了该小鼠模型在抗结 直肠肿瘤药物研究中的应用和意义。

关键词: APC 基因; Min 小鼠; Wht 基因

\section{Use of $A p c(\mathrm{Min} /+)$ mouse model in the studies of intestinal tumors}

\author{
SHENG Hong-Qiang, CHEN Jian, LAI Mao-De \\ Department of Pathology and Pathophysiology, School of Medicine, Zhejiang University, Hangzhou 310058, China
}

\begin{abstract}
More than thirty kinds of mutant or knockout mice bearing intestinal neoplasm have been reported up to the present. Apc(Min/+) mouse holding multiple intestinal neoplasia, provides an appropriate model to evaluate human familial adenomatous polyposis. APC is an important tumor-suppressor gene in the Wnt pathway, which is involved in the pivotal signal transduction cascade in animal embryogenesis and colorectal carcinogenesis. Apc(Min/+) mouse model was presented as aspects of the strain background, genotype/phenotype, divergent canonical Wnt signaling pathway, methylation of tumor-suppressor gene, TGF- $\beta$ signaling pathway and multidrug resistance gene, etc. This review also introduced the application and signification of the mouse model in studies of anti-colorectal tumor drugs.
\end{abstract}

Keywords: APC; Min mouse; Wnt

家族性腺瘤性息肉病(familial adenomatous polyposis, FAP)病例最早在 1721 年由 Menzel 报道, 随后 1882 年 Harrison 揭示了其遗传特征, 1925 年 Lockhart 进一步证实了这是一种癌前病 变, 遗传符合孟德尔定律。FAP 患者主要临床 症状是腹痛、腹泻、便血和肠梗阻, 最明显的特 点是多发性结直肠腺瘤性息肉。FAP 患者的肠 道息肉若不加治疗, 40 岁时一个或数个息肉发
生癌变的机率可达 $100 \%$ 。FAP 可分为 “经典” 的家族性腺瘤性息肉病、Gardner 综合征、 Turcot 综合征、轻表型家族性腺瘤性息肉病等 亚类吕它们均是 APC(adenomatous polyposis coli)基因突变所致的常染色体显性遗传病。 Min(multiple intestinal neoplasia)小鼠具有肠道 多发性腺瘤特征, 是目前公认的较为理想的 FAP 研究模型。 


\section{Min 基因突变小鼠模型的历史}

1990 年美国 Wisconsin-Madison 大学 Dove 实 验室在世界上首次篮选出了 $A p c(\mathrm{Min} /+)$ 小鼠, 使得 人类对 FAP 的研究有了良好的模型动物 ${ }^{[2]}$ 。 C57BL/6J 雄性小鼠接受突变剂 ENU (ethylnitrosourea)处理后与雌性小鼠 AKR/J 杂交后, 研究者发 现有些子代容易发生肠道肿瘤, 进一步研究揭示此 子代小鼠 $A p c$ 基因两条链中其中一条链的第 850 号 密码子发生无义突变(nonsense mutation), 编码亮氨 酸的密码子(TTG)转变成终止密码子(TAG), 形成截 断蛋白。这种小鼠由于 $A p c$ 基因第 850 号密码子等 位突变(allele mutation)引起肠道多发性腺瘤, 因而 被称之为 Min(multiple intestinal neoplasia)小鼠。自 $A p c(\mathrm{Min} /+)$ 小鼠诞生以来, 陆续有研究人员制备出 和 $A p c$ 基因高频突变位点有关的突变小鼠和基因敲 除小鼠 (knockout mice), 如 $\Delta 716, \Delta 1309$ 和 $1638 \mathrm{~N}^{[2 \sim 5]}$, 其中 $A p c{ }^{\Delta 716}$ 敲除小鼠比经典的 $A p c(\mathrm{Min} /+)$ 突变小鼠形成更多的小肠微小息肉, 每 只小鼠平均达到 300 个, 后者一般只有 30 个㟶。需 要明确的是 $A p c(\mathrm{Min} /+)$ 突变小鼠特指 $A p c$ 基因第 850 号密码子出现无义突变 $(\mathrm{TTG} \rightarrow \mathrm{TAG}$ ) 的 $\mathrm{C} 57 \mathrm{BL} / 6 \mathrm{~J}$ 品系小鼠, 其它 $A p c$ 基因突变或敲除的小 鼠即使出现肠道多发性腺瘤也不宜称之为 $\operatorname{Apc}(\mathrm{Min} /+)$ 突变小鼠。

目前也有许多靶向敲除的小鼠如 Msh2 敲除小

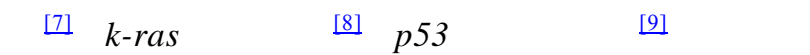
将几种突变或敲除小鼠的后代杂交繁殖, 形成新品 系的突变-敲除小鼠, 用来研究几种不同蛋白同时发 生失活和/或激活的效应。迄今, 和肠道肿瘤相关的 基因突变小鼠或敲除小鼠大约有 30 多种 ${ }^{[10]}$, 这些小 鼠的出现, 大多是受了 $A p c(\mathrm{Min} /+)$ 突变小鼠研究的 启发, 还有新的基因突变或敲除小鼠不断出现, 它 们为肠道肿瘤的深层机制探讨提供良好的实验载 体。Apc(Min/+)小鼠模型在国内的称呼有 $\mathrm{Min}$ 基因 突变小鼠、 $\mathrm{Min}$ 小鼠等, 英文常见表达有 $A p c(\mathrm{Min} /+)$ mouse、Apc $c^{\mathrm{Min} /+}$ mouse、 $A p c^{\mathrm{Min}} / A p c^{+}$mouse 和 $\mathrm{C} 57 \mathrm{BL} / 6 \mathrm{~J}-A p c^{M i n} / \mathrm{J}$ 等。据我们所知国内很少有实验 室在从事 Min 小鼠相关研究。

2 Min 基因突变小鼠模型的分子遗传学与 表型特征

结直肠癌主要有 3 大类: 散发性结直肠癌 (sporadic CRC) 、遗传性非息肉病性结直肠癌 (hereditary nonpolyposis colorectal cancer, HNPCC) 和 FAP，后两者的遗传度约为 $5 \% \sim 15 \%$ 。目前认为错 配修复基因(mismatch repair gene, MMR)缺陷是 HNPCC 的典型机制; APC- $\beta$-catenin-TCF 主导的 Wnt 通路失调是 FAP 发生的主要途径。APC 基因是 $\mathrm{Wnt}$ 途径中重要的抑癌基因, 该途径不仅是动物胚胎发 育过程中关键的信号转导途径 ${ }^{[11]}$, 也对肿瘤的发生 发展起到不同寻常的作用 ${ }^{[12]}$ 。诸多研究支持结直肠 癌的发生发展是多因素、多基因、多阶段的过程。 散发性结直肠癌不仅涉及到 MMR, 而且与 Wnt 通 路的 $A P C$ 基因突变关系密切, HNPCC 也被发现 $A P C$ 和 $\beta$-catenin 呈高频突变 ${ }^{[13 ~ 15]}$ 。人类抑癌基因 $A P C$ 定位于 5q21-22, 开放读码框 $8538 \mathrm{bp}$, 含有 15 个外 显子, 基因产物由 2843 个氨基酸残基组成。其中 $A P C$ 第 15 外显子非常大, 包含 $6571 \mathrm{bp}$, 突变也主 要集中在第 15 外显子 ${ }^{[16]}$ 。大量研究表明多于 $75 \%$ 的已知突变落于第 15 外显子的突变成簇区 (mutation cluster region, MCR)密码子 1 284 1 580。我们以往 的文章也介绍了 $\beta$-catenin 如何参与结直肠肿瘤发病 过程 ${ }^{[17,18]}$ 。由此可见, 抑癌基因 $A P C$ 的失活可能在 结直肠肿瘤启动中起到了“扳机”作用, APC 基因也 一直是结直肠癌研究的热点和重点。

小鼠 $A p c$ 基因定位在第 18 号染色体，与人类 $A P C$ 基因有 $90 \%$ 的同源 19$]$ 。 Min 小鼠主要的遗传 学特征是 $A p c$ 基因两条链中的一条链第 850 号密码 子发生无义突变, 即第 2549 号碱基发生颠换 $(\mathrm{T} \rightarrow \mathrm{A})$, 编码亮氨酸的密码子 (TTG) 转变成终止密码子 (TAG), 从而造成截断蛋白, 抑癌基因 $A p c$ 不能有效 地起作用。近年来还发现了在 Min 小鼠中具有调节 肿瘤数量的遗传位点: Mom1、Mom2、Mom7 等位 点, 其中 Mom1 定位于 4 号染色体远端与人类染色 体 1p35 36 高度同源, 而在人类结直肠癌中该区域 是常有杂合性缺失的一个区域。有意思的是部分 携带有 Mom2 基因的 Min 小鼠具有抑制肠道肿瘤的

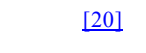

Min 小鼠肠道肿瘤容易出现 $A p c$ 杂合性缺失, 即 $A p c$ 等位基因中正常的那条 $A p c^{+}$缺失, 即使在微 腺瘤(为腺瘤早期肉眼不易发现的病变)中也可发 现。最近 Yamada 等 ${ }^{[21]}$ 对老年 Min 小鼠的研究发现, 显微镜下小肠微腺瘤较少而大小不一(平均直径 1 $286.96 \pm 1069.78 \mu \mathrm{m}$ ); 大肠微腺瘤较多而体积小(平 均直径 $176.04 \pm 410.84 \mu \mathrm{m})$ 。而且这些微腺瘤具有 
$\beta$-catenin 免疫染色阳性的特点。但是肉眼可见的肿 瘤在小肠段比大肠段高发, 两者平均数分别是 30 60 个/小鼠和 0 5 个/小鼠。经杂合性缺失(LOH) 分析, 这些来自 Min 小鼠的大肠微腺瘤和大肠肿瘤 一样都呈现了 $A p c^{+}$的等位缺失, 仅保留了 $A p c^{M i n}$ 条 带, 这符合 Knudson's 的两次打击学说。另外, 从肉 眼可见肿瘤和微腺瘤在 Min 小鼠小肠段和大肠段的 分布差异特点来看, $A p c$ 基因杂合性缺失在小肠和 大肠肿瘤发生中所起的作用是有差异的。微腺瘤也 呈现出肿瘤的 $A p c^{+}$的等位缺失的特点, 然而微腺瘤 数目远远大于肿瘤数目的事实, 说明要发展成肿瘤, 仅有 $\mathrm{Apc}^{+}$的等位缺失也是不够的, 可能还需要其它 因素参与。作者参与发展了一种新型动物模型或许 可以用来解释这一差异。经炎症诱发剂葡聚糖硫酸 钠(dextran sodium sulfate, DSS)诱导, 可以在 Min 小 鼠大肠处快速形成肿瘤(4 周以内)。该模型的肿瘤也 证实了 $A p c$ 基因杂合性缺失的特点 $(100 \%, 40 / 40)$, 有意思的是, 我们检测了 $k$-ras 第一外显子, $\beta$-catenin 的第三外显子, 均没有提示突变 $(0 \%$, $0 / 40)$ 。另外在 DSS 的参与下, Min 小鼠的大肠肿瘤 无论从形成数量还是速度上, 均比单独的末受任何 处理的 Min 小鼠要来的明显。

Min 小鼠自然生存期一般不超过 120 天，往往 伴随着慢性贫血特征, 表现为网织红细胞增多、红 细胞计数减少。研究者最早在没有可靠的分子检测 技术条件时, 利用贫血特质这个比较简单的方法来 䇴选 $A p c(\mathrm{Min} /+)$ 小鼠(准确率几乎可以达到 100\%); 纯合子的 $A p c(\mathrm{Min} / \mathrm{Min})$ 小鼠非常容易死亡, 难以繁 殖, 有些在母鼠子宫内就因不能正常发育而流产死 亡。Min 小鼠还常见黑便、脱肛、脾肿大以及高脂 血症等表现。另外, 部分雌性 Min 小鼠可形成乳腺 肿瘤。最近 Tomita 等 ${ }^{[22]}$ 报道在致癌剂 AOM 的处理 下 Min 小鼠容易发生胃癌。因此, Min 小鼠若加以 修饰可能是对多种疾病进行研究的良好模型。

\section{Min 基因突变小鼠模型与肠道肿瘤}

\subsection{Min 小鼠模型与 Wnt 途径}

正常细胞 Wnt 途径通过 APC 蛋白, 糖原合成酶 激酶 $-3 \beta(\mathrm{GSK}-3 \beta)$ 轴蛋白或传导蛋白 (axin 或 conductin) 等与 $\beta$-catenin 结合形成多蛋白复合体降 解 $\beta$-catenin。若上述一种或一种以上成分的异常就 有可能引起细胞 Wnt 途径表达的异常。比如, APC
基因第 15 外显子的突变成簇区有 7 个重复序列, 每 个重复序列含有 20 个氨基酸, 截断蛋白 APC 至少 需要保留 3 个重复序列才能有效降解 $\beta$-catenin。另 外， $\beta$-catenin 基因突变多发生在外显子 3 的 33 、

$37 、 41$ 或者 45 号密码子, 其缺失或突变使得 $\beta$-catenin 不能与 GSK-3 $\beta / A P C$ 结合而磷酸化, 因而 不能被有效降解, 导致胞浆内大量非磷酸化的 $\beta$-catenin 蓄积, 进入细胞核与转录因子 TCF 相互作 用，调控下游基因如 c-myc、c-jun、PPAR- $\mathrm{Y}$ 、 cyclinD1、MMP-7、connexin43 和 Cdx1 等表达。

随着 Min 小鼠为主的模型动物的深入研究, 发 现了 Wnt 途径与结直肠肿瘤间许多更有意思的联 系。在人类中由于 $A P C$ 基因突变可能导致结直肠肿 瘤、FAP、Gardner 综合征和 Lynch 综合征等, 但是 几乎不形成小肠的多发性息肉; 而 $A p c$ 突变的 Min 小鼠模型中, 大肠、小肠均可见的多发性肿瘤, 同时 在小肠处有大量肉眼可见多发腺瘤性息肉(注: 人类 FAP 指的是大肠的家族性腺瘤性息肉病)。其它的 $A p c$ 突变小鼠以及 $A p c^{\Delta 716}$ 敲除小鼠和 $\mathrm{Min}$ 小鼠一样, 发生的息肉主要集中在小肠。但是当 $A p c^{\Delta 716}$ 敲除小 鼠和 $C d \times 2^{+/}$突变小鼠杂交时, 形成的子代 $A p c^{\Delta}$ ${ }^{716} C d x 2^{+/-}$小鼠中发生的息肉则主要在结肠中 ${ }^{[23]}$ 。目 前已知正常状况下 $C d x 2$ 参与了肠道形态发育、分化, 并且可以抑制大肠的息肉和肿瘤, 可能由于突变的 $C d x 2$ 基因通过多功能激酶 mTOR 途径影响了细胞 增殖和凋亡, 以及染色体的不稳定, 进而影响息肉 的发生发展, 但是 $A p c$ 基因与 $C d x 2$ 基因之间是通过 一个什么样的机制平衡, 使得 $A p c^{\Delta{ }^{716}} C d x 2^{+/}$小鼠的 息肉频发在结肠, 还是一个令人费解的谜。

Husoy 等 ${ }^{[24]}$ 在细胞水平研究表明 $A p c^{M i n}$ 突变降 低了连接蛋白 $(\mathrm{Cx} 43)$ 介导的缝隙连接细胞间交流 GJIC (gap junctional intercellular communication)以 及微管极化, 但是不影响 $\beta$-catenin 以及下游基因 E-cadherin 的定位和表达, 这表明 $A p c$ 基因仅仅是一 条链的突变, 就足以影响微管极化水平, 但是可能 不会引起 Wnt 途径失调, 对后者而言 $A p c$ 等位基因 两条链的失活可能是必需的。

\section{$3.2 \mathrm{Min}$ 小鼠模型与甲基化}

抑癌基因 $\mathrm{CpG}$ 岛甲基化导致抑癌基因失活和转 录抑制是导致肿瘤发生的重要原因之一 ${ }^{[25]}$ 。目前认 为基因启动子区域高甲基化状态阻止了基因转录因 子与启动子结合。高甲基化的 $\mathrm{CpG}$ 岛是特异的甲基 
化结合蛋白 (methyl-CpG-binding protein, $\mathrm{MeCP}$ )与 $\mathrm{HDAC}$ (组蛋白去乙酰基酶)协同作用的结果。 $\mathrm{MeCP}$ 复合体由甲基化 $\mathrm{CpG}$ 岛结合区(methyl-CpG-binding domain, MBD)和转录抑制区(transcriptional repression domain, TRD)构成, Mbd2 是 CDKN2A (cyclin-dependent kinase inhibitor-2A, 或称为 $\mathrm{p} 16^{\mathrm{Ink} 4 \mathrm{~A}}$ ) 甲基化 $\mathrm{CpG}$ 岛的结合性抑制剂。Apc $\mathrm{Ain}^{\mathrm{i} /+} \mathrm{Mbd} 2^{\text {- }}$ 小 鼠模型 ${ }^{[26]}$ 实验表明, $M b d 2$ 的缺失可能使抑癌基因 p16 得以正常转录翻译, 从而抑制或降低肠道肿瘤 的发生。甲基转移酶 Dnmt1 是另一个和甲基化相关 的重要基因, 其表达增高可能促进抑癌基因的甲基 化。Apc ${ }^{\mathrm{Min} / \mathrm{t}} \mathrm{Dnmt} 1^{-/+}$小鼠模型提示 Dnmt1 缺陷引起 的甲基化 $\mathrm{CpG}$ 岛的降低可以抑制肠道息肉形成 227$]$

\section{$3.3 \mathrm{Min}$ 小鼠模型与 MMR 缺陷和抑癌基因 $p 53$ 的} 失活

错配修复基因缺陷和抑癌基因 $p 53$ 的失活是结 直肠肿瘤发生的另外两个重要机制。在人类遗传性 非息肉病性结直肠癌(HNPCC)中, 目前发现的错配 修复基因主要有 6 种, 分别是 hMSH2、hMSH3、 GTBP、hMLH1、hPMS1 和 hPMS2, 其中只有 hMSH2 或 hMLH1 的突变在绝大多数 HNPCC 中可见, 其余 的与之相关性不太明显。我们以往的数据 ${ }^{[28]}$ 显示即 使在结直肠癌的早期即腺瘤阶段就可以表现出由于 错配修复缺陷导致的微卫星不稳定性。Sohn 等 [29] 的研究表明, 由于 $M s h 2$ 的敲除, 在 $A p c^{M i n /+} M s h 2^{\text {/- }}$ 小鼠的肠道肿瘤中, $p 53$ 基因的 Exon5 8 的突变率达 到了 $45.5 \%(5 / 11$ 例), 而对照组 Min 小鼠的肠道肿 瘤中未观察到 $p 53$ 的突变 $(0 / 8$ 例)。对 $A p c$ 基因第 15 外显子的分析结果是, $A p c^{\mathrm{Min} /+} \mathrm{Msh} 2^{-/}$小鼠的肠道单 个肿瘤 $A p c$ 基因突变的位点数平均高达 10 个, 携带 有 $A p c$ 截断突变的肿瘤占 $82 \%(9 / 11$ 例 $)$; 相应的对照 组 $A p c^{\mathrm{Min} /+}$ 小鼠的肠道单个肿瘤 $A p c$ 基因突变的位 点数平均只有 1.8 个, 未发现肿瘤内有 $A p c$ 截断突变 $(0 / 8$ 例)。换言之, Min 小鼠中 Msh2 的缺陷可以增强 $A p c$ 基因和 $p 53$ 基因的突变, 导致抑癌基因的失活, 从而诱发大量的肠道肿瘤。

\subsection{Min 小鼠模型与 TGF- $\beta$ 途径}

已知人类结直肠肿瘤中, TGF- $\beta$ 通路中的 $\mathrm{Smad} 2$ 和 $\mathrm{Smad} 4$ 作为抑癌基因起着重要的作用。小 鼠 Smad2 和 Smad4 毗邻, 与 $A p c$ 基因一起位于 18 号染色体, 也被认为对肠道肿瘤的发生有着重要作 用。Apc ${ }^{\Delta 716 /+} S m a d 4{ }^{+/}$小鼠中提示, Smad4 的无义突
变促进了 $A p c^{\Delta 716 /+{ }^{5}}$ 除小鼠肠道肿瘤的发展 ${ }^{[30]}$; 但 是在 $A p c^{\Delta 716 /+} S m a d 2^{+/-}$小鼠中, 经 $\mathrm{LOH}$ 分析证实新 生息肉已经出现 $A p c$ 和 $S m a d 2$ 的杂合性缺失, Smad2 的无义突变并不影响 $A p c^{\Delta 716 /+}$ 小鼠小肠息肉的发生 发展 ${ }^{[31]}$ 。类似的, $A p c^{\mathrm{Min} /+} \mathrm{Dcc}{ }^{+/}$小鼠也反映了 $\mathrm{Dcc}$ 的 无义突变不影响肠道息肉发生 ${ }^{[32]}$ 。

\subsection{Min 小鼠模型与多药耐药基因 1(MDR1)}

临床上认为肿瘤细胞多药耐药是导致肿瘤化学 治疗失败的关键因素之一, $A p c^{\mathrm{Min} /+} M d r 1 \mathrm{a} / \mathrm{b}^{-/-}$小鼠模 型置换了 $A p c^{M i n /+}$ 小鼠中的 $M d r 1$ 基因, 形成 $M d r 1$ 的缺陷，阻止多药耐药 $(M d r)$ 基因的表达,进而抑制 该类型小鼠的息肉发生 ${ }^{[33]}$ 。因此该模型从反面证实 抑制多药耐药基因的表达有助于息肉的减少, 这为 临床解决化疗失败提供了新的思路。由于多药耐药 基因 1(MDR1)的启动子位置的部分编码序列与 $\beta$-catenin 与 TCF4 连接区的结构域编码序列相一致 的, 因此 MDR1 也被认为是 Wnt 途径调控的重要靶 位之一。失活的 Wnt 通路就可能激活多药耐药基因 启动子, 使得 MDR1 表达增加促进肿瘤的发展。

\subsection{Min 小鼠模型与其他相关基因}

流行病学调查提示高脂血症、肥胖和结直肠肿 瘤发生有较为紧密的关系, 因此有研究者利用一些 基因修饰小鼠如高血脂小鼠 $L d l r^{t m 1 H e r}$ 、肥胖小鼠 $L e p r^{o b}$ 、Lepr $r^{d b}$ 小鼠与遗传性缺陷的肠道肿瘤模型小 鼠配合以研究高脂血症、肥胖等因素和结直肠肿瘤 之间的关系。还有报道证实表皮生长因子受体 EGFR(epidermal growth factor receptor)缺陷可以抑 制大肠肿瘤的发生, EGFR 酪氨酸激酶抑制剂处理 后也能抑制 Min 小鼠的肠道肿瘤生长 ${ }^{[34]}$ 。另外, 功 能性 Fas 配体缺失将增强 Min 小鼠肠道肿瘤的发 生 ${ }^{[35]}$ 。最新研究 ${ }^{[36]}$ 提示 $E p h B$ 表达下降可以加速 Min 小鼠肠道肿瘤发生，并形成具有侵袭性的腺癌，因 此 $E p h B$ 表达被认为是结直肠癌发生过程中的重要 步骤。

4 Min 小鼠模型在抗结直肠肿瘤药物研究 中的应用

药物干预 Min 小鼠，一方面可以揭示药物的抗 肿瘤属性, 而且 Min 小鼠模型也是一种有力的抗结 直肠肿瘤药物篮选工具; 另一方面特殊的药物作用 靶位也可以解释可能的肿瘤发生途径。不少文献提 供了药物作用于 Min 小鼠模型后,出现小鼠结直肠肿 
瘤相关基因表达差异和/或肿瘤的发生率的差异等。 Rao 等 ${ }^{[37]}$ 报道了对苯二亚甲基硒腈 $(1,4$ phenylenebisselenocyanate, p-XSC)可以明显降低 Min 小鼠肠 道肿瘤的发生, 而且抑制了 $\beta$-catenin 和环氧化酶 $(\mathrm{COX})-2$ 的表达, 因为通常 $\beta$-catenin 和 Cox-2 的高 表达可以促进肿瘤的发生。Cox-2 选择性抑制剂 Celecoxib 也能抑制 Min 小鼠的肠道肿瘤 ${ }^{[38]}$ 。Peuter 等 ${ }^{[39,40]}$ 的研究提示阿斯匹林(aspirin)则通过诱导调 亡和降低前列腺素 E2(PGE2)来抑制 Min 小鼠的肿 瘤发生。Niho 等 ${ }^{[411}$ 发现通过过氧化物酶体增生物激 活受体(PPAR- $\gamma$ )抑制剂吡咯列酮(pioglitazone)治疗 可以抑制 $A p c^{\Delta 1309}$ 小鼠的肠道肿瘤以及高脂血症, 而不少研究证实 PPAR- $\gamma$ 在肿瘤内的表达增高。

Mahmoud 等 ${ }^{[42]}$ 将咖啡酸苯乙酯 CAPE(caffeic acid phenethyl ester)作用于 Min 小鼠, 观察其对肿瘤的预 防作用。结果发现, 在饲料中添加 $0.15 \%$ 的 CAPE 可使肿瘤生成下降 $63 \%$ 。进一步观察肠内组织, 证 实 CAPE 所起到的肿瘤预防作用与增加肠上皮细胞 的凋亡和抑制增殖有关。CAPE 还可降低 Min 小鼠 肠内细胞 $\beta$-catenin 的表达。更多肠道肿瘤药物相关 作用信息可以访问 Chemoprevention of Colorectal Cancer 站点 http://www.inra.fr/。

\section{参考文献(References):}

[1] Foulkes WD. A tale of four syndromes: familial adenomatous polyposis, Gardner syndrome, attenuated APC and Turcot syndrome. Qjm, 1995, 88(12): 853-863.

[2] Moser AR, Pitot HC, Dove WF. A dominant mutation that predisposes to multiple intestinal neoplasia in the mouse. Science, 1990, 247(4940): 322-324. [DOI]

[3] Oshima M, Oshima H, Kitagawa K, Kobayashi M, Itakura C, Taketo M. Loss of Apc heterozygosity and abnormal tissue building in nascent intestinal polyps in mice carrying a truncated Apc gene. Proc Natl Acad Sci USA, 1995, 92(10): 4482-4486. [DOI]

[4] Quesada CF, Kimata H, Mori M, Nishimura M, Tsuneyoshi T, Baba S. Piroxicam and acarbose as chemopreventive agents for spontaneous intestinal adenomas in APC gene 1309 knockout mice. Jpn J Cancer Res, 1998, 89(4): 392-396.

[5] Fodde R, Edelmann W, Yang K, van Leeuwen C, Carlson C, Renault B, Breukel C, Alt E, Lipkin M, Khan PM, Kucherlapati R. A targeted chain-termination mutation in the mouse Apc gene results in multiple intestinal tumors. Proc Natl Acad Sci USA, 1994, 91(19): 8969-8973. [DOI]

[6] Moser AR, Dove WF, Roth KA, Gordon JI. The Min (multiple intestinal neoplasia) mutation: its effect on gut epithelial cell differentiation and interaction with a modifier system. $J$
Cell Biol, 1992, 116(6): 1517-1526. [DOI]

[7] Reitmair AH, Redston M, Cai JC, Chuang TC, Bjerknes M, Cheng H, Hay K, Gallinger S, Bapat B, Mak TW. Spontaneous intestinal carcinomas and skin neoplasms in Msh2-deficient mice. Cancer Res, 1996, 56(16): 3842-3849.

[8] Johnson L, Greenbaum D, Cichowski K, Mercer K, Murphy E, Schmitt E, Bronson RT, Umanoff H, Edelmann W, Kucherlapati R, Jacks T. K-ras is an essential gene in the mouse with partial functional overlap with $\mathrm{N}$-ras. Genes Dev, 1997, 11(19): 2468-2481. [DOI]

[9] Donehower LA, Harvey M, Slagle BL, McArthur MJ, Montgomery CA, Jr., Butel JS, Bradley A. Mice deficient for p53 are developmentally normal but susceptible to spontaneous tumours. Nature, 1992, 356(6366): 215-221. [DOI]

[10] Boivin GP, Washington K, Yang K, Ward JM, Pretlow TP, Russell R, Besselsen DG, Godfrey VL, Doetschman T, Dove WF, Pitot HC, Halberg RB, Itzkowitz SH, Groden J, Coffey RJ. Pathology of mouse models of intestinal cancer: consensus report and recommendations. Gastroenterology, 2003, 124(3): 762-777. [DOI]

[11] Cadigan KM, Nusse R. Wnt signaling: a common theme in animal development. Genes Dev, 1997, 11(24): 3286-3305. [DOI]

[12] Morin PJ, Weeraratna AT. Wnt signaling in human cancer. Cancer Treat Res, 2003, 115: 169-187.

[13] Brueckl WM, Limmert T, Brabletz T, Guenther K, Jung A, Hermann K, Wiest GH, Kirchner T, Hohenberger W, Hahn EG, Wein A. Mismatch repair deficiency in sporadic synchronous colorectal cancer. Anticancer Res, 2000, 20(6C): 4727-4732.

[14] Kim JC, Koo KH, Roh SA, Cho YK, Kim HC, Yu CS, Kim HJ, Kim JS, Cho MK. Genetic and epigenetic changes in the APC gene in sporadic colorectal carcinoma with synchronous adenoma. Int J Colorectal Dis, 2003, 18(3): 203-209.

[15] Akiyama Y, Nagasaki H, Yagi KO, Nomizu T, Yuasa Y. Beta-catenin and adenomatous polyposis coli (APC) mutations in adenomas from hereditary non-polyposis colorectal cancer patients. Cancer Lett, 2000, 157(2): 185-191. [DOI]

[16] Hampton GM, Ward JR, Cottrell S, Howe K, Thomas HJ, Ballhausen WG, Jones T, Sheer D, Solomon E, Frischauf AM, Bodmer WF. Yeast artificial chromosomes for the molecular analysis of the familial polyposis APC gene region. Proc Natl Acad Sci USA, 1992, 89(17): 8249-8253. [DOI]

[17] LAI Mao-De. Molecular mechanism of carcinogenesis in colorectal cancer. Journal of Practical Oncology, 2000, 15(2): 73-78.

来茂德. 大肠癌发生的分子机理. 实用肿瘤杂志, 2000 , 15(2): 73-78.

[18] LAI Mao-De. Action of $\beta$-catenin and its correlated Factors in carcinogenesis of colorectal cancer (CRC). Chinese Journal of Clinical and Experimental Pathology, 2000, 16(5): 412-414.

来茂德. $\beta$-catenin 及其相关因子在结直肠癌发生中的 作用. 临床与实验病理学杂志, 2000, 16(5): 412-414. 
[19] Su LK, Kinzler KW, Vogelstein B, Preisinger AC, Moser AR, Luongo C, Gould KA, Dove WF. Multiple intestinal neoplasia caused by a mutation in the murine homolog of the APC gene. Science, 1992, 256(5057): 668-670. [DOI]

[20] Silverman KA, Koratkar R, Siracusa LD, Buchberg AM. Identification of the modifier of Min 2 (Mom2) locus, a new mutation that influences Apc-induced intestinal neoplasia. Genome Res, 2002, 12(1): 88-97. [DOI]

[21] Yamada Y, Hata K, Hirose Y, Hara A, Sugie S, Kuno T, Yoshimi N, Tanaka T, Mori H. Microadenomatous lesions involving loss of Apc heterozygosity in the colon of adult Apc(Min/+) mice. Cancer Res, 2002, 62(22): 6367-6370.

[22] Tomita H, Yamada Y, Oyama T, Hata K, Hirose Y, Hara A, Kunisada T, Sugiyama Y, Adachi Y, Linhart H, Mori H. Development of gastric tumors in Apc(Min/+) mice by the activation of the beta-catenin/Tcf signaling pathway. Cancer Res, 2007, 67(9): 4079-4087. [DOI]

[23] Aoki K, Tamai Y, Horiike S, Oshima M, Taketo MM. Colonic polyposis caused by mTOR-mediated chromosomal instability in Apc+/Delta716 Cdx2+/- compound mutant mice. Nat Genet, 2003, 35(4): 323-330. [DOI]

[24] Husoy T, Cruciani V, Knutsen HK, Mikalsen SO, Olstorn HB, Alexander J. Cells heterozygous for the ApcMin mutation have decreased gap junctional intercellular communication and connexin43 level, and reduced microtubule polymerization. Carcinogenesis, 2003, 24(4): 643-650. [DOI]

[25] ZHANG Li-Li, WU Jian-Xin. DNA methylation: an epigenetic mechanism for tumorigenesis breeds. Hereditas (Beijing), 2006, 28(7): 880-885.

张丽丽, 吴建新. DNA 甲基化一一肿瘤产生的一种表观 遗传学机制. 遗传, 2006, 28(7): 880-885.

[26] Sansom OJ, Berger J, Bishop SM, Hendrich B, Bird A, Clarke AR. Deficiency of Mbd2 suppresses intestinal tumorigenesis. Nat Genet, 2003, 34(2): 145-147. [DOI]

[27] Eads CA, Nickel AE, Laird PW. Complete genetic suppression of polyp formation and reduction of $\mathrm{CpG}$-island hypermethylation in Apc(Min/+) Dnmt1-hypomorphic mice. Cancer Res, 2002, 62(5): 1296-1299.

[28] CHENG Lei, WANG Hui-Ping, HUANG Qiong, LAI Mao-De. Microsatellite instability and relative gene expressions in sporadic and familial adenomatous polyposis adenomas. Hereditas (Beijing), 2004, 26(1): 1-7.

程蕾, 王慧萍, 黄琼, 来茂德. 结直肠腺瘤的微卫星不 稳定状态与相关基因表达的研究. 遗传, 2004, 26(1): 1-7.

[29] Sohn KJ, Choi M, Song J, Chan S, Medline A, Gallinger S, Kim YI. Msh2 deficiency enhances somatic Apc and p53 mutations in Apc+/-Msh2-/- mice. Carcinogenesis, 2003, 24(2): 217-224. [DOI]

[30] Takaku K, Oshima M, Miyoshi H, Matsui M, Seldin MF, Taketo MM. Intestinal tumorigenesis in compound mutant mice of both Dpc4 (Smad4) and Apc genes. Cell, 1998, 92(5): 645-656. [DOI]
[31] Takaku K, Wrana JL, Robertson EJ, Taketo MM. No effects of Smad2 (madh2) null mutation on malignant progression of intestinal polyps in Apc(delta716) knockout mice. Cancer Res, 2002, 62(16): 4558-4561.

[32] Fazeli A, Dickinson SL, Hermiston ML, Tighe RV, Steen RG, Small CG, Stoeckli ET, Keino-Masu K, Masu M, Rayburn H, Simons J, Bronson RT, Gordon JI, TessierLavigne M, Weinberg RA. Phenotype of mice lacking functional Deleted in colorectal cancer (Dcc) gene. Nature, 1997, 386(6627): 796-804. [DOI]

[33] Yamada T, Mori Y, Hayashi R, Takada M, Ino Y, Naishiro Y, Kondo T, Hirohashi S. Suppression of intestinal polyposis in Mdr1-deficient ApcMin/+ mice. Cancer Res, 2003, 63(5): 895-901.

[34] Goodlad RA, Ryan AJ, Wedge SR, Pyrah IT, Alferez D, Poulsom R, Smith NR, Mandir N, Watkins AJ, Wilkinson RW. Inhibiting vascular endothelial growth factor receptor-2 signaling reduces tumor burden in the ApcMin/+ mouse model of early intestinal cancer. Carcinogenesis, 2006, 27(10): 2133-2139. [DOI]

[35] Fingleton B, Carter KJ, Matrisian LM. Loss of functional Fas ligand enhances intestinal tumorigenesis in the Min mouse model. Cancer Res, 2007, 67(10): 4800-4806. [DOI]

[36] Batlle E, Bacani J, Begthel H, Jonkheer S, Gregorieff A, van de Born M, Malats N, Sancho E, Boon E, Pawson T, Gallinger S, Pals S, Clevers H. EphB receptor activity suppresses colorectal cancer progression. Nature, 2005, 435(7045): 1126-1130. [DOI]

[37] Rao CV, Cooma I, Rodriguez JG, Simi B, El-Bayoumy K, Reddy BS. Chemoprevention of familial adenomatous polyposis development in the $\mathrm{APC}(\mathrm{min})$ mouse model by 1 , 4-phenylene bis(methylene)selenocyanate. Carcinogenesis, 2000, 21(4): 617-621. [DOI]

[38] Jacoby RF, Seibert K, Cole CE, Kelloff G, Lubet RA. The cyclooxygenase- 2 inhibitor celecoxib is a potent preventive and therapeutic agent in the min mouse model of adenomatous polyposis. Cancer Res, 2000, 60(18): 5040-5044.

[39] Reuter BK, Zhang XJ, Miller MJ. Therapeutic utility of aspirin in the ApcMin/+ murine model of colon carcinogenesis. BMC Cancer, 2002, 2: 19. [DOI]

[40] Barnes CJ, Lee M. Chemoprevention of spontaneous intestinal adenomas in the adenomatous polyposis coli Min mouse model with aspirin. Gastroenterology, 1998, 114(5): 873-877. [DOI]

[41] Niho N, Takahashi M, Kitamura T, Shoji Y, Itoh M, Noda T, Sugimura T, Wakabayashi K. Concomitant suppression of hyperlipidemia and intestinal polyp formation in Apc-deficient mice by peroxisome proliferator-activated receptor ligands. Cancer Res, 2003, 63(18): 6090-6095.

[42] Mahmoud NN, Carothers AM, Grunberger D, Bilinski RT, Churchill MR, Martucci C, Newmark HL, Bertagnolli MM. Plant phenolics decrease intestinal tumors in an animal model of familial adenomatous polyposis. Carcinogenesis, 2000, 21(5): 921-927. [DOI] 